УДК 35А 35, 47A58, 65N13, 65F10, 76W05

\title{
WAVELETS IN THE TRANSPORT THEORY OF HETEROGENEOUS REACTING SOLUTES
}

\author{
C. C A T T A N I*, E. L A S ER R A** \\ * Dipartimento di Matematica "G.Castelnuovo", Univ. di Roma "La Sapienza", ** Dipartimento di \\ Matematica e Informatica, Univ. di Salerno, Italia
}

Получено 03.04.2002

\begin{abstract}
In this paper we consider the one-dimensional convection (advection) dispersion equation of the transport theory of heterogeneous reacting solutes in porous media. A wavelet solution is in the framework of multi-resolution analysis.

Рассматривается дисперсионное уравнение одномерной конвекции (адвекции) теории транспорта гетерогенных реагируюших растворенных веществ в пористых средах. Вейвлетное решение получено в рамках мультирезольвентного аналиюа.

Ровглядається дісперсійне рівняння одновимірної конвекції (адвекціi) теорії транспорту гетерогенних реагуючих ровчинених речовин у пористих середовищах. Вейвлетний ровв'яэок одержано в рамках мультиревольвентного аналіву.
\end{abstract}

\section{INTRODUCTION}

The transport theory of reacting solutes in a porous medium is considered under the hypotheses that the motion of solute transport is unidirectional, isothermal and devoid of instabilities; it takes place in a heterogeneous porous medium and the content, the density and the viscosity of the water in the medium are constant during the process. Since we are mainly interested in the chemical reaction equations we assume that the physical parameters defining the medium are unaffected by the transport, leaving the size of the pores, their distribution in the solid (and so on) unchanged. The chemical species defining the solid are at rest (while the chemical species defining the solute are mobile) in the medium, so that the mathematical model of the transport solution under chemical reactions (source-free) is the following algebraic-differential system $[6](\Theta, D, Q$ constants $)$

$$
\left\{\begin{array}{l}
\Theta \frac{\partial c_{i}}{\partial t}=L c_{i} \\
L \stackrel{\text { def }}{=} D \frac{\partial^{2}}{\partial x^{2}}-Q \frac{\partial}{\partial x} \\
f_{r}\left(c_{i}, \frac{\partial c_{i}}{\partial t}\right)=0, \quad(i=1, \ldots, \# P ; r=1, \ldots)
\end{array}\right.
$$

also called the convection-dispersion system. System (1) is a differential and algebraic system in the unknown \#P functions $c_{i}(x, t)$, \#P being the number of tenads [?] involved in the chemical reaction. The algebraic equations (1) 3 express the relationships among concentrations of reaction participants, to be fulfilled irrespective of the contributions of the individual processes (both chemical and/or physical) influencing the concentrations $c_{i}$.

\section{ONE DIMENSIONAL SOLUTE TRANSPORT EQUATION}

In a one-dimensional domain $\Omega \subset \mathcal{R}$ let $x$ be the coordinate, $I$ a finite interval of time $t,(I \stackrel{\text { def }}{=}$ $\{t: 0<t<T, T<\infty\})$. We consider a sufficiently fast and reversible reaction of heterogeneous type [6], where the medium's original solution consists of two reacting cations $M_{1}, M_{3}$ in equilibrium with a cation exchanger ${ }^{1} \bar{M}_{e}$. The displacing solution contains a reacting cation $M_{1}$ and a non-reacting anion $M_{2}$. The transport affecting reaction is represented by the chemical reactions (for binary cation exchange)

$$
M_{1}+\overline{M_{3} M_{e}} \rightleftharpoons M_{3}+\overline{M_{1} M_{e}}
$$

There are four tenads in the system, three reacting $M_{1}, M_{3}, M_{e}$ and one chemical non-reacting species $M_{2}$. The basic equations (1) for the concentrations

1 Dissolved in water and solid phase transport participants are given symbols $M$ and $\bar{M}$, respectively. 
$c_{1}, \bar{c}_{1}, c_{2}, c_{3}, \bar{c}_{3}$ are $[6$, p. 1237$]$

$$
\left\{\begin{array}{c}
\Theta \frac{\partial c_{1}}{\partial t}+\rho \frac{\partial \bar{c}_{1}}{\partial t}=L c_{1}, \\
\Theta \frac{\partial c_{2}}{\partial t}=L c_{2}, \\
\Theta \frac{\partial c_{3}}{\partial t}+\rho \frac{\partial \bar{c}_{3}}{\partial t}=L c_{3}, \\
\rho \frac{\partial \bar{c}_{1}}{\partial t}+\rho \frac{\partial \bar{c}_{3}}{\partial t}=0 \\
K_{13}=\frac{\bar{c}_{1} c_{3}}{c_{1} \bar{c}_{3}}
\end{array}\right.
$$

with $K_{13}$ a given constant. The $e$ subscript, representing $\bar{M}_{e}$, has been dropped from the $\bar{c}$ functions, for them $\rho$ is the anologous of $\Theta$ and represents the porous medium's bulk density (mass of the medium's solids/the medium's volume). In the heterogeneous reactions both the liquid and solid phases are involved in the tenads. From $(2)_{4}$ we get

$$
\bar{c}_{1}+\bar{c}_{3}=c
$$

since the exchange capacity does not vary with $x$. Then it follows:

$$
\Theta \frac{\partial}{\partial t}\left(c_{1}+c_{3}\right)=L\left(c_{1}+c_{3}\right)
$$

that, with the change of variable $u=c_{1}+c_{3}$, becomes the linear equation

$$
\Theta \frac{\partial u}{\partial t}=L u,
$$

whose solution can be expressed in terms of wavelets [2]. Deriving the condition (2) 5 with respect to $t$ and taking into account equation (3) we obtain from $(2)_{1,4}$ the following nonlinear system [6]:

$$
\begin{gathered}
\left(\Theta+\frac{\rho p_{i}}{g}\right) \frac{\partial c_{i}}{\partial t}-\frac{\rho p_{i}}{g} \frac{\partial c_{j}}{\partial t}=L c_{i} \quad i, j=1,3 \\
p_{i} \stackrel{\text { def }}{=} K_{13}\left(c-c_{i}\right), \quad g \stackrel{\text { def }}{=} K_{13} c_{1}+c_{3},
\end{gathered}
$$

from which we obtain the non-linear equation

$$
a \frac{\partial g}{\partial t}=g L g, \quad L \stackrel{\text { def }}{=} D \frac{\partial^{2}}{\partial x^{2}}-Q \frac{\partial}{\partial x}
$$

with a suitable constant $a$. In particular, we restrict ourselves to the following one-dimensional initialboundary problem:

$$
\begin{gathered}
\frac{\partial g}{\partial t}=g L g, \quad L \stackrel{\text { def }}{=} D \frac{\partial^{2}}{\partial x^{2}}-Q \frac{\partial}{\partial x}, \quad(a=1) \\
\left\{\begin{array}{lll}
u(x, 0)=u_{B}(x), & 0 \leq x \leq 1, & t=0, \\
u(0, t)=0, & x=0, & t>0, \\
u(1, t)=0, & x=1, & t>0
\end{array}\right.
\end{gathered}
$$

and we assume as the initial function

$$
\begin{gathered}
u_{B}(x)=\left\{\begin{array}{l}
1, \quad x \in \Lambda_{1}, \\
-1, \quad x \in \Lambda_{-1}, \\
0, \quad x \notin \Lambda_{1} \cup \Lambda_{-1},
\end{array}\right. \\
\Lambda_{1} \stackrel{\text { def }}{=}\left\{x: 0<x_{0} \leq x \leq x_{1}<1\right\}
\end{gathered}
$$

which corresponds to the realistic case of an impulse function when $\left|x_{2}-x_{0}\right| \rightarrow 0$.

\section{MULTI-RESOLUTION ANALYSIS IN HAAR \\ BASIS}

The Haar scaling function $\Phi_{k}^{n}(x) \stackrel{\text { def }}{=} 2^{n / 2} \Phi\left(2^{n} x-k\right)$ has a compact support on the dyadic interval

$$
D_{k}^{n} \stackrel{\text { def }}{=}\left[\frac{k}{2^{n}}, \frac{k+1}{2^{n}}\right)
$$

where its value is 1 . The Haar family of wavelets

$$
\Psi_{k}^{n}(x) \stackrel{\text { def }}{=}\left\{2^{\frac{n}{2}} \Psi\left(2^{n} x-k\right)\right\}_{k, n \in Z}
$$

is a complete orthonormal system for the $L_{2}(\mathcal{R})$ functions [5]

$$
\Psi_{k}^{n}(x)=\left\{\begin{array}{l}
1, \quad x \in\left[\frac{k}{2^{n}}, \frac{k+1 / 2}{2^{n}}\right) \\
-1, \quad x \in\left[\frac{k+1 / 2}{2^{n}}, \frac{k+1}{2^{n}}\right) \\
0, \quad \text { elsewhere. }
\end{array}\right.
$$

Let $V_{n}, n \in Z$ be the subspace of $L_{2}(\mathcal{R})$ defined as the set of the piecewise constant functions $f(x)$ of compact support on $D_{k}^{n}$ ( $n$ fixed), and $W_{n}$ the orthogonal subspace such that the axioms of multiresolution (or multiscale) analysis [4, 5] are fulfilled,

$$
\left\{\begin{array}{l}
L_{2}(\mathcal{R})=\bigoplus_{n \in Z} W_{n}=V_{q} \oplus \bigoplus_{j \geq q} W_{j}, \quad q \in Z \\
V_{n+1}=V_{n} \oplus W_{n}
\end{array}\right.
$$

being $\oplus$ the direct sum of orthogonal spaces. The set of functions $\left\{\Psi_{k}^{n}\right\} \quad(n \in Z)$ represents an orthonormal basis for $L_{2}(\mathcal{R})$.

Fixing the resolution value $N<\infty$, in (8), the $L_{2}(\mathcal{R})$ space is approximated by $L_{2}(\mathcal{R}) \cong \bigoplus_{n=0}^{N} W_{n}$, that is,

$$
f(x) \cong \pi^{N+1} f(x) \stackrel{\text { def }}{=} \alpha_{0}^{0}+\sum_{n=0}^{N} \sum_{k=0}^{n} \beta_{k}^{n} \Psi_{k}^{n}(x),
$$



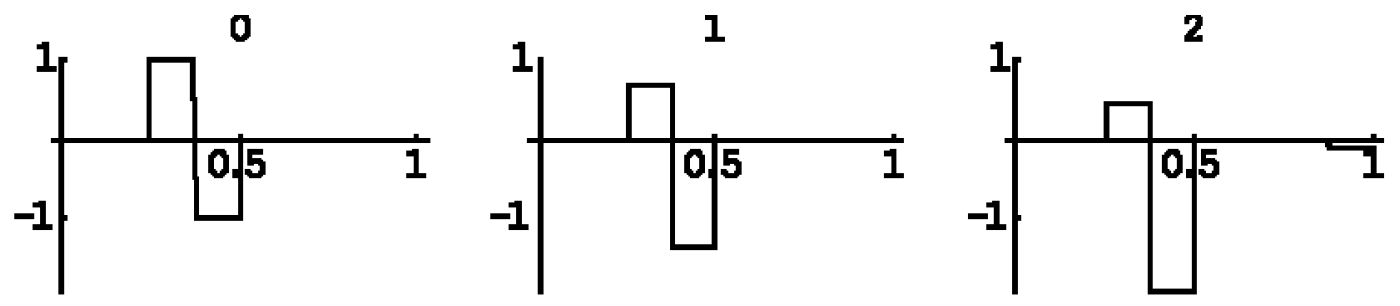

Рис. 1. Haar-wavelet representation of the wave solution

being $\pi^{n}$ a projection operator into $V_{n+1}$, so that $\pi^{n}: L_{2}(\mathcal{R}) \rightarrow V_{n+1}$

Choosing a number of dyadic nodes $x_{k} \stackrel{\text { def }}{=} \frac{k}{2^{n}},\left(k=0, \ldots, 2^{n}-1\right)$ the dyadic discretization is the operator $\nabla^{n}: L_{2}(\mathcal{R}) \rightarrow L_{2}\left(Z\left(2^{-n}\right)\right)$, with $L_{2}\left(Z\left(2^{-n}\right)\right) \subset L_{2}(\mathcal{R})$ being the set of $L_{2}(\mathcal{R})$ functions sampled at $x_{k}$. The action of $\nabla^{n}$ on $f(x)$ is such that $\nabla^{n} f(x)=\mathbf{f}^{n}$ with $\mathbf{f}^{n}=\left\{f_{0}, f_{1}, \ldots, f_{2^{n}-1}\right\}$ and $\left\{\left.f_{k} \stackrel{\text { def }}{=} f(x)\right|_{x=x_{k}}, 0 \leq k \leq 2^{n}-1\right\}$. The fast Haarwavelet transform $\mathcal{H}$ of $\mathbf{f}^{N}$ is the linear operator $[1$, $4]$

$$
\begin{aligned}
& \mathcal{H}: L_{2}\left(Z\left(2^{-n}\right)\right) \rightarrow V_{n} \mid \mathbf{f}^{N} \mapsto \mathcal{H} \mathbf{f}^{N}= \\
& =\left\{\alpha_{0}^{0}, \beta_{k}^{n}\right\}_{k=0, \ldots, n}^{n=0, \ldots, 2^{N}-1},
\end{aligned}
$$

where $\alpha_{0}^{0}, \beta_{k}^{n}$ are the wavelet coefficients.

According to the above definitions, the projection operator $\pi^{n}: L_{2}(\mathcal{R}) \rightarrow V_{n+1}$ is factorized as $\pi^{n}=$ $\mathcal{H} \nabla^{n}$.

A $p$-order Cardinal spline, is a $C^{p-1}([0,1))$ differentiable operator

$\mathcal{S}^{p}: L_{2}\left(Z\left(2^{-N}\right)\right) \rightarrow C^{p-2}([0,1)): \mathbf{f}^{N} \mapsto s(x) \stackrel{\text { def }}{=} \mathcal{S}^{p} \mathbf{f}^{N}$

such that for the differential operator

$$
L: L_{2}\left(Z\left(2^{-N}\right)\right) \rightarrow L_{2}\left(Z\left(2^{-N}\right)\right)
$$

it is

$$
L \mathcal{H}=\mathcal{H} L \mathcal{S}^{p}
$$

There follows that, given the set $\mathbf{f}^{N}$ and computed the spline of a sufficiently large order, the splinederivative of $\mathcal{H} \mathbf{f}^{N}$ belongs to the same space of $\mathbf{f}^{N}$ $[1,4]$.

According to the above, using splines and wavelets up to the resolution $N$, the approximate solution of the equation (4)is the vector $\mathbf{u}^{N}\left(\in V_{N+1}\right)$, i.e. assuming the Euler formula for the time-derivative

$$
\pi^{N} \frac{\partial u}{\partial t}=\frac{\mathbf{u}^{N+1}-\mathbf{u}^{N+1}}{\Delta t}
$$

we have from (4)

$$
\mathbf{u}^{N+1}=\mathbf{u}^{N}+\Delta t L\left(\mathcal{H} \mathbf{u}^{N}\right)
$$

and, according to (11)

$$
\mathbf{u}^{N+1}=\left(1+\Delta t \mathcal{H} L \mathcal{S}^{p}\right) \mathbf{u}^{N}
$$

With the boundary condition (5), time step $\Delta t=$ 0.01 , and assuming in (4) $Q=D=1$ and in (5) $x_{0}=\frac{1}{4}, x_{1}=\frac{3}{8}, x_{2}=\frac{1}{2}$, after 3 time steps $(t=0.05)$ we obtain the evolving function of Fig.

1. C. Cattani Haar Wavelet Spline // Journal of Interdisciplinary Mathematics.- 2001.- vol.4, No. 1.P. 35-47.

2. C.Cattani and E.Laserra, // Transport Theory of Homogeneous Reacting Solutes.- Ukrainian Mathematical Journal.- 2001.- P. vol. 53, No. 8.1048-1052

3. C.Cattani and F.Passarella Nonsimple Porous materials with Thermal Relaxation // Rend. Ist. Lombardo Acc. di Scienze e Lett.,A.- 1996.- vol. 130, No 2.P. 1-19.

4. C.Cattani and M.Pecoraro Non Linear Differential Equations in Wavelet Basis // Acoustic Bulletin.2000.- vol. 3, (4).- P. 4-10.

5. I.Daubechies Ten lectures on wavelets/CBMS-NSF Regional Conference Series in Applied Mathematics.Philadelphia.:SIAM, 1992.

6. J.Rubin Transport of Reacting Solutes in Porous Media: Relation Between Mathematical Nature of Problem Formulation and Chemical Nature of Reactions // Water Resour. Res.- 1983.- No 13.- P. 12311252.

7. D.Schweich and M.Sardin Absorption, partition, ion exchange, and chemical reaction in batch reactors or in columns // J. Hydrol.- No 50.- 1981.- P. 1-33. 